\title{
Conditions for disembarkation of Caenorhabditis japonica from its carrier insect Parastrachia japonensis
}

\author{
Etsuko Okumura ${ }^{1,2}$, Ryusei Tanaka ${ }^{1}$ and Toyoshi Yoshiga ${ }^{1,2, *}$
}

\begin{abstract}
Bacteriophagous Caenorhabditis japonica forms a phoretic and necromenic association with the burrower bug Parastrachia japonensis. C. japonica dauer larvae (DL) disembark from the body surface of $P$. japonensis females before their propagation in the nest of the carrier insect. In this study, we examined the conditions and possible cues for disembarkation. In high humidity conditions, not all the DL that were naturally associated with or artificially loaded on P. japonensis disembarked from their carriers. The presence of food bacteria or the soil habitat did not enhance disembarkation. However, the presence of $\mathrm{P}$. japonensis nymphs promoted disembarkation. These results indicate that only some $\mathrm{C}$. japonica DL leave their carriers under conditions of high humidity and that the presence of nymphs after hatching stimulates disembarkation. DL collected from $\mathrm{P}$. japonensis females several months after eclosion showed gravitactic behavior while those from $P$. japonensis 3 days after loading did not, suggesting behavioral changes in DL during their association with the carrier. Nematol. Res. 43(1), 1-7 (2013).

Key words: dauer, entomophilic, gravitaxis, necromeny, phoresy
\end{abstract}

\section{INTRODUCTION}

Phoresy is one of the associations occurring between nematodes and insects. Phoretic nematodes use insects as a vector for transfer between reproductive sites (Poiner, 1975). Necromeny is a special type of phoresy in which nematodes on the insect carrier wait for the death of the carrier and develop by feeding on bacteria present on the carrier's decomposing corpse (Sudhaus and Schulte, 1989). Many different types of nematodes have phoretic associations with different insect species (Poiner, 1975; Kiontke, 2006); however, studies on the mechanisms of embarkation and disembarkation by phoretic nematodes are limited (Kiontke, 1996; Baird, 1999).

Caenorhabditis japonica Kiontke, Hironaka \& Sudhaus is a bacterial-feeding nematode found on the burrower bug Parastrachia japonensis Scott (K iontke et al., 2002). C. japonica dauer larvae ( $\mathrm{DL}$ ) are detectable throughout the year as clusters on the body surface of the insect, especially under the scutellum of most female P. japonensis, except those exhibiting provisioning behavior; they are however rare on male $P$. japonensis. DL is nonfeeding and a developmental diapause stage, which is specialized for dispersal and survival in unfavorable conditions. Our observations showed that $C$. japonica use $P$. japonensis as a vehicle for transfer to reproductive sites within the nest of its carrier

${ }^{1}$ Laboratory of Nematology, Department of A pplied Biological Sciences, Faculty of A griculture, Saga University, Saga 840-8502, Japan

2 The United Graduate School of A gricultural Sciences, Kagoshima University, Kagoshima 890-8580, Japan

* Corresponding author, e-mail: tyoshiga@cc.saga-u.ac.jp insect, where it can propagate on egg carcasses and nymphal cadavers, thus suggesting a phoretic and necromenic association (Okumura et al., 2013a). The host insect $P$. japonensis is a monophagous and gregarious hemipteran insect with a unique biology (T sukamoto and Tojo, 1992). P. japonensis spends most of the year aggregating and forming clusters on green leaves during their reproductive diapause. They mate in March, and during July, the gravid $P$. japonensis females lay their eggs as an egg mass in a nest within the leaf litter. The mother insects carry an egg mass that protects the eggs from predators and microbes, and they provision the nest with drupes from the deciduous tree Schoepfia jasminodora Sieb. et Zucc. after hatching.

In a previous study, we demonstrated that $\mathrm{C}$. japonica found on female $P$. japonensis resume their mobility in high humidity conditions, when they disembark and propagate in the nests of P. japonensis (Okumura et al., 2013a). In the present study, we tested for possible disembarkation cues in C. japonica DL. We also compared the behavioral changes in $\mathrm{C}$. japonica $\mathrm{DL}$ during their association with $\mathrm{P}$. japonensis. U pward movement of $C$. japonica DL was observed during nematode culture (T anaka et al., 2010a), which suggested negative gravitaxis in C. japonica, although $\mathrm{DL}$ on the carrier insect had to move downward from their carrier. We hypothesized that the gravitactic behavior of $\mathrm{C}$. japonica changed during the association, and thus, we compared the gravitactic behavior of $\mathrm{DL}$ and its possible role in disembarkation. 


\section{MATERIALS AND METHODS}

Nematodes and insects:

Caenorhabditis japonica strain $\mathrm{H} 1$ was isolated from a $P$. japonensis female collected in Hinokuma Mountain Prefectural Park, Kanzaki City, Saga Prefecture, Japan. C. japonica DL were maintained on dog food agar medium (Hara et al., 1981) seeded with Escherichia coli strain OP50 at $25^{\circ} \mathrm{C}$. Phoretically active $\mathrm{DL}$ were collected as described by T anaka et al. (2010a). In brief, to collect DL, a sterile yel-

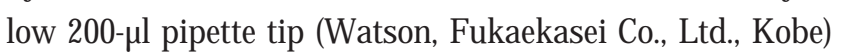
was vertically placed in dog food agar in a 100-ml culture bottle. Nictating $D L$ on the top of the tip were picked up with a fine needle and washed three times with distilled water before use.

Reproductive diapause females of $P$. japonensis were collected in Hinokuma (October-December 2008 and March 2009) and were used for experiments.

Caenorhabditis japonica DL were artificially loaded on P. japonensis, as described by T anaka et al. (2010b). In short, nematode-free reproductive diapause female $P$. japonensis were prepared by partly soaking in tap water for 3 days and rehydrated nematodes on the insects were washed off. A fter 24-hr incubation of a reproductive dia- pause female of $P$. japonensis with approximately $1,000 \mathrm{C}$. japonica DL in a $6-\mathrm{cm}$ plastic petri dish, the $\mathrm{P}$. japonensis loaded with $C$. japonica DL were placed in a desiccator with $97 \%$ relative humidity (RH) for 3 days and then in a desiccator with $85 \% \mathrm{RH}$ for 1 day at $25^{\circ} \mathrm{C}$ to obtain quiescent $\mathrm{DL}$ with a condition similar to field conditions. $\mathrm{K}_{2} \mathrm{SO}_{4}$ - and $\mathrm{KCl}$-saturated solutions were used to produce $97 \%$ and $85 \% \mathrm{RH}$ conditions at $25^{\circ} \mathrm{C}$, respectively, according to Winston and Bates (1960). A fter the desiccation treatments, we used $P$. japonensis in the disembarkation experiments.

Disembarkation of $C$. japonica from its carrier:

We placed a single $P$. japonensis female into a plastic cup (diameter $=7 \mathrm{~cm}$ at the bottom and $10.3 \mathrm{~cm}$ at the top, depth $=4.3 \mathrm{~cm}$ ), and the insect was covered with a short stainless steel pipe (diameter $=7.2 \mathrm{~cm}$, height $=3.4 \mathrm{~cm}$ ), the top end of which was covered with wire netting (pore size $=$ $1 \mathrm{~mm}^{2}$ ). A piece of wet Kimwipe S-200 (Kimberly-Clark Co., Kulesia, T okyo) was placed at the bottom to maintain high humidity (Fig. 1A). The plastic cup was covered with a lid and placed in a large plastic box $(23 \times 16 \times 8.5 \mathrm{~cm})$ with a wet Kimtowel (Kimberly-Clark Co., Kulesia, Tokyo) to maintain high humidity in the box. Nematodes that disembarked from insects were collected by washing the
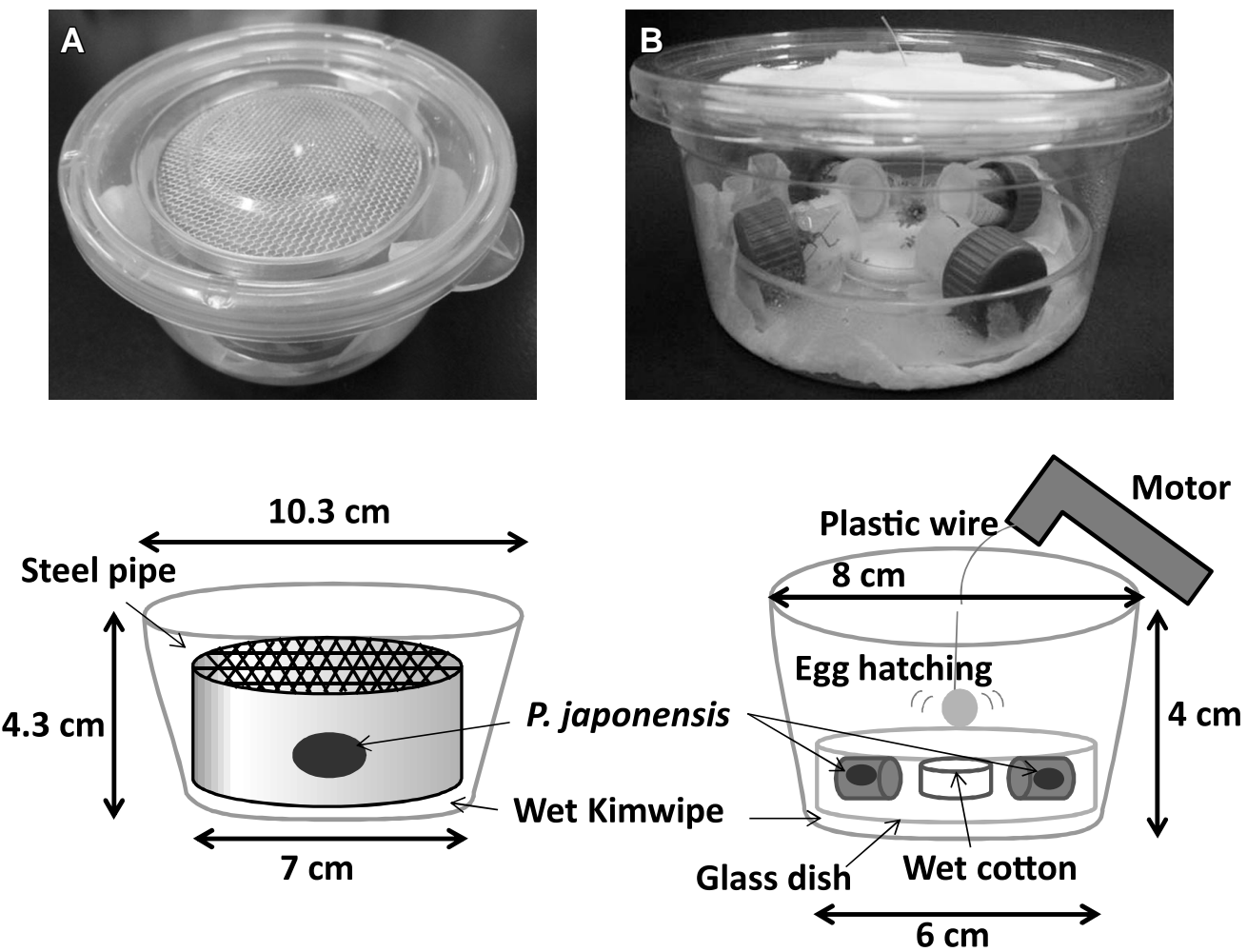

Fig. 1. Systems used for the disembarkation experiments. A, equipment used for the disembarkation experiment. A Parastrachia japonensis female was placed in a steel pipe in the plastic cup. The plastic cup was used for most experiments, except the hatching experiment. B, equipment used for the hatching experiment. F our or five tubes each containing a female $P$. japonensis were placed in the cup. A $n$ egg mass was hung by a nylon fishing line from the center of the lid, and synchronized hatching was stimulated intermittently by motor vibration for $20 \mathrm{~min}$. 
Kimwipe and small plastic cup at 24-hr intervals for 13 days for the naturally associated condition because $P$. japonensis hatching occurs about 12 days after oviposition in the field. Collection of nematodes from the artificially loaded condition was stopped after 10 days because nematode disembarkation was not observed 8 days after starting the experiment. The piece of Kimwipe was replaced each day. A fter 13 days, the insects were dissected and placed in a $6-\mathrm{cm}$ Syracuse watch glass with tap water for $24 \mathrm{hr}$ to separate the nematodes from the insects. The nematodes that remained on the insects were counted using a stereomicroscope. The percentage of disembarkation was calculated based on the total number of nematodes that disembarked from an insect and nematodes remaining on the insect.

To test for possible bacterial disembarkation cues, a 3$\mathrm{cm}$ plate containing nematode growth medium (NGM) (Liuzzi et al., 2012) with a fresh E. coli OP50 lawn was also placed in the stainless steel pipe in the plastic cup (Fig. 1A). The total numbers of disembarked nematodes were calculated 13 and 10 days after starting the experiments for naturally associated and artificially loaded P. japonensis, respectively.

To study disembarkation in soil, we filled a plastic cup (diameter $=3 \mathrm{~cm}$ at the bottom and $4.2 \mathrm{~cm}$ at the top, depth $=2.4 \mathrm{~cm}$ ) with soil collected from the P. japonensis habitat. A few drops of water were added to the cup to maintain high humidity, and a single female P. japonensis naturally associated with C. japonica was placed in the cup. A fter covering with a lid, the cup containing the single female insect was placed in a high humidity plastic box $(23 \times 16$ $\times 8.5 \mathrm{~cm}$ ) with the lid side of the cup facing down. The plastic box was incubated at $25^{\circ} \mathrm{C}$. T en days later, we counted the number of $C$. japonica in the soil and on the $P$. japonensis. The nematodes in the soil were separated using the Baermann funnel technique, and the number of $C$. japonica was counted using a stereomicroscope. We then calculated the proportion of $C$. japonica that disembarked from their carrier. In a preliminary experiment, we confirmed the absence of $C$. japonica in the soil.

We used the system shown in Fig. $1 \mathrm{~B}$ to evaluate the effect of $P$. japonensis hatching on disembarkation. Reproductive diapause females of $P$. japonensis that are naturally associated with $C$. japonica were incubated individually in 100\% RH for 10-14 days in a plastic cup (diameter $=7 \mathrm{~cm}$ at the bottom and $10.3 \mathrm{~cm}$ at the top, depth $=4.3$ $\mathrm{cm}$ ) before starting the experiment. The nematodes that disembarked during this incubation period were collected and counted using a stereomicroscope each day. A single female was placed in a plastic tube (diameter $=1.5 \mathrm{~cm}$, length $=3$ $\mathrm{cm}$ ) with one side sealed with a nylon mesh (pore size $=5$ $\mu \mathrm{m})$ and the other side capped. A small piece of wet Kimwipe was placed inside the tube. Four or five tubes were placed in a plastic cup (diameter $=6 \mathrm{~cm}$ at the bottom and $8 \mathrm{~cm}$ at the top, depth $=4 \mathrm{~cm}$ ) with wet cotton wool at the center. A mature egg mass with the surface partly covered by symbiotic bacteria provided for the P. japonensis by the mother insect was hung in the center. Synchronized hatching was stimulated intermittently by motor vibration for 20 min, which mimicked the behavior of the mother $P$. japonensis. Synchronized hatching was observed from 10 min after starting the vibration. Nematodes that disembarked from insects during hatching were collected by washing the tubes and Kimwipes about 30 to $90 \mathrm{~min}$ after stopping the vibration, depending on the hatching conditions.

A fter the hatching experiment, individual P. japonensis were placed in plastic cups with high humidity for another 12 days. Nematodes that disembarked during the 12-day incubation period were collected and counted. Next, we placed a tube containing P. japonensis and two 3rd instar nymphs in a plastic cup in high humidity conditions for 24 $\mathrm{hr}$. A fter exposure to the nymphs for $24 \mathrm{hr}, \mathrm{P}$. japonensis were dissected and each placed in a 6-cm Syracuse watch glass with $10 \mathrm{ml}$ of tap water for $24 \mathrm{hr}$ to release the nematodes from the insects. The nematodes were counted using a stereomicroscope. We calculated the number of nematodes that disembarked during 10-14 days in 100\% humidity before starting the experiment and the total number of nematodes during this period.

Negative gravitaxis experiments:

Parastrachia japonensis females collected in October and December 2008, and M arch 2009 were immediately used for experiments. The P. japonensis females artificially loaded with C. japonica DL were kept for 3 days at $25^{\circ} \mathrm{C}$ after desiccation treatment and then used for experiments. $C$. japonica DL were picked up from $P$. japonensis using a nematode picker and were soaked in $M 9$ buffer to revive them from their quiescent state. A bout $20 \mathrm{DL}$ were inoculated onto the center of a 9-cm NGM plate. A fter the water around the DL was absorbed by the NGM plate and they started moving, we set the plate in a vertical position. A fter $1 \mathrm{hr}$, we counted the number of nematodes that moved upward or downward from the inoculation point. The negative gravitaxis index was calculated as follows: (the number of nematodes moving in an upward direction - the number of nematodes moving in a downward direction)/total number of inoculated nematodes (Okumura et al., 2013c). Nematodes $\pm 1 \mathrm{~cm}$ from the central line were excluded from the dataset. 
Statistical analysis:

A NOVA was used for analyses of disembarkation and negative gravitaxis experiments. Values of negative gravitaxis index were compared using a Bonferroni/Dunn test. All statistical analyses were performed using StatView Ver. 4.54 (A bacus Concepts, Inc., New Jersey).

\section{RESULTS}

Effect of high humidity on the disembarkation of $\mathrm{C}$. japonica DL from their carrier insects:

Reproductive diapause P. japonensis females with naturally associated $C$. japonica $D L$ on their body surface placed in $100 \% \mathrm{RH}$ conditions; the DL resumed their mobility, although not all the nematodes left their carriers within 13 days (Fig. 2). Over $70 \%$ of the nematodes disembarked from three of 10 insects, whereas $<50 \%$ of the associated nematodes disembarked from the other seven insects. Less than $60 \%$ of the nematodes left the insects during the incubation period after artificial loading.

Effect of bacteria on the disembarkation of C. japonica DL from their carrier insects:

To determine the possible disembarkation cues other than high humidity, we used a small NGM plate with an E. coli lawn as a food cue. Many nematodes remained on the carrier insects in the presence of $\mathrm{E}$. coli, and the percentages of nematodes that disembarked from the insects in the presence or absence of $E$. coli were not significantly different in naturally associated nematodes and those grown on E. coli

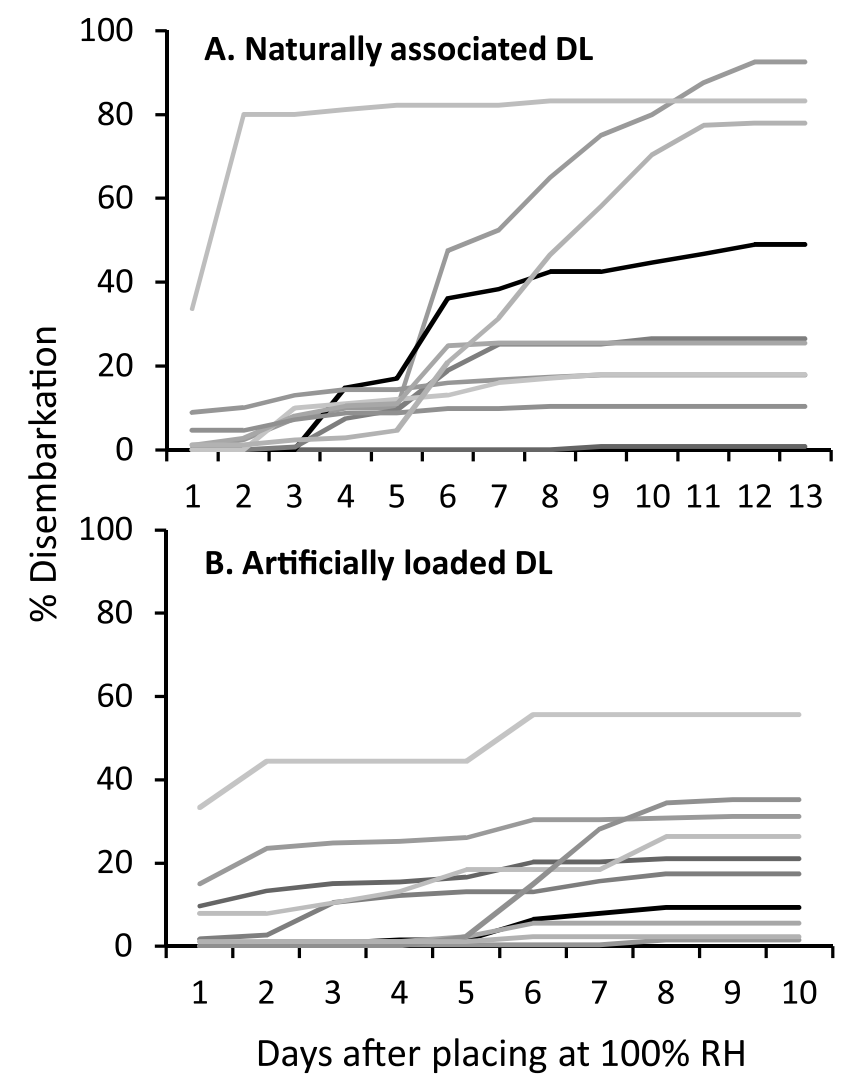

Fig. 2. Disembarkation profiles of Caenorhabditis japonica dauer larvae (DL) from their $P$. japonensis carriers in high humidity conditions. Reproductive diapause females that naturally associated with $C$. japonica and artificially loaded females were exposed to $100 \%$ humidity (Fig. 1A), and the daily disembarkation rate was recorded. Each line indicates the accumulated percentage of disembarked nematodes from a single $P$. japonensis. T en insects were used for each condition.

\section{A. Naturally associated DL}

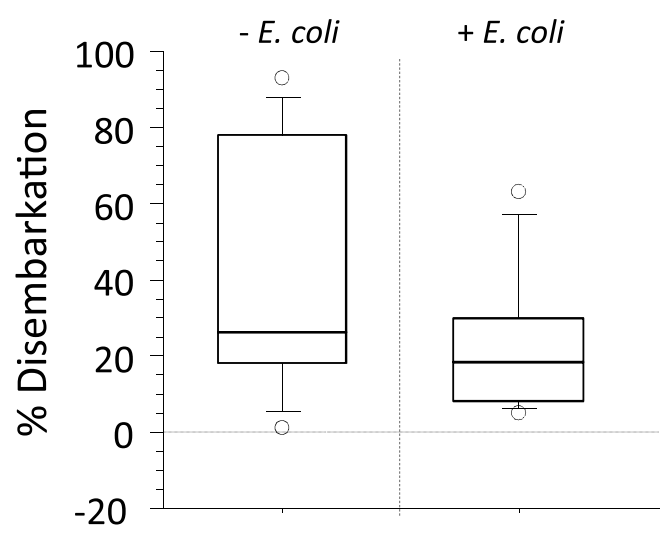

\section{B. Artificially loaded DL}

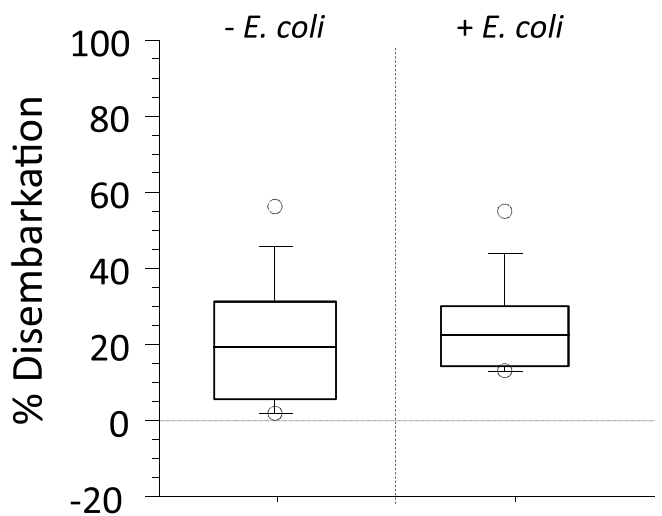

Fig. 3. Effect of bacteria on disembarkation. Escherichia coli strain OP50-containing culture medium was placed in a plastic cup (Fig. IA) with reproductive diapause $P$. japonensis females. T en $P$. japonensis females that were naturally associated or artificially loaded with $C$. japonica DL were used in each experiment. The total numbers of disembarked nematodes were calculated 13 and 10 days after starting the experiments using naturally associated and artificially loaded DL, respectively. The line inside the box plot is the median, the top of the box is the third quartile, the bottom of the box is the first quartile, the upper bar is the 90th percentile, the lower bar is the 10th percentile, and the white circle is an outlier. Bars indicate SD. There were no statistical differences $(A, P=0.2450 ; B, P=0.6350$, A NOVA). 
that were artificially loaded onto insects $(P=0.245$ and 0.635, respectively, A NOVA) (Fig. 3). Fresh soil from the C. japonica habitat also failed to stimulate disembarkation, and only $24 \%$ of $C$. japonica DL disembarked from their carrier insects within 10 days of starting the experiments (data not shown).

Effect of hatching and nymphs on C. japonica DL disembarkation from their carrier insects:

Before hatching, a mean of $7.7 \%$ (minimum $=1.8 \%$, maximum $=15.4 \%$ ) of $D L$ left the $P$. japonensis during the 14-day incubation period in high humidity conditions. Very few nematodes disembarked in response to the $P$. japonensis hatching stimuli (Fig. 4). A fter hatching, the P. japonensis were placed in 100\% RH conditions for a further 12 days, but very few nematodes (maximum $=7 \mathrm{DL}$ ) disembarked during this period. In contrast, when $P$. japonensis females were incubated for 12 days and exposed to nymphs, significantly higher percentages $(6.1 \%-76.3 \%)$ of DL disembarked from the carrier insects within $24 \mathrm{hr}$, compared to the 14-day incubation and hatching stimuli $(P<$ $0.01, \mathrm{P}<0.0001$, respectively, A NOV A) (Fig. 4).

Changes in the gravitactic behavior of $C$. japonica DL:

We compared the gravitactic behavior of $C$. japonica DL collected from P. japonensis in different conditions (Fig. 5). C. japonica DL exhibited upward movement, and their mean NGI values were positive from 3 days after artificial loading. In contrast, significantly larger numbers of nematodes on insects collected in the field during October, December, and March moved downward on an NGM plate $(P<0.001$, A NOVA ). The NGI values for October, December, and $M$ arch were negative and significantly lower than that of 3 days after artificial loading.

\section{DISCUSSION}

Caenorhabditis japonica DL are phoretically associated with their carrier insect $P$. japonensis females, and they have to leave their carrier insect for their propagation. In a previous study, we demonstrated that $C$. japonica DL disembarked and propagated in $\mathrm{P}$. japonensis nests (Okumura et al., 2013a). High humidity is a necessary condition for the resumption of nematode mobility, but there may be cues for disembarkation. In this study, we examined possible disembarkation cues in the form of soil, food bacteria, P. japonensis hatching, and $P$. japonensis nymphs. The presence of nymphs was the most effective cue for disembarkation by C. japonica DL. We also found that the duration of the association with the insect affected the gravitactic behavior. These results suggest that high humidity with the presence

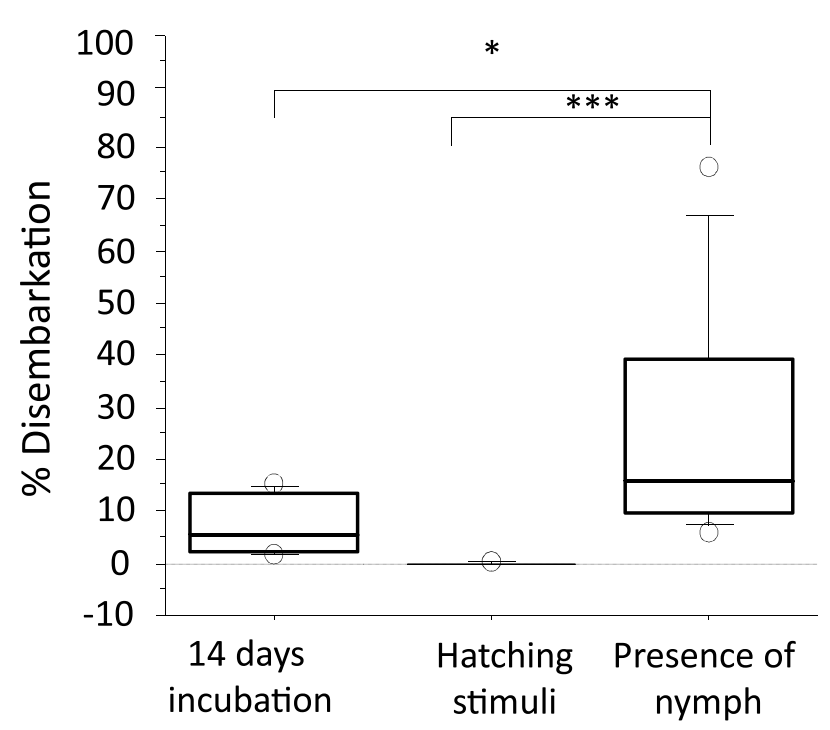

Fig. 4. Comparison of C. japonica DL disembarkation from P. japonensis in different conditions. The extent of disembarkation during the first 14 days in the presence of high humidity condition, hatching, and 3rd instar $\mathrm{P}$. japonensis larvae were compared $(N=9)$. P. japonensis females were initially incubated in high humidity conditions for 14 days to remove $\mathrm{DL}$ before being exposed to hatching stimuli. A fter being exposed to the hatching stimuli, the insects were incubated for another 12 days in high humidity conditions (Fig. $1 \mathrm{~A}$ ) and then exposed to nymphal stimuli (Fig. 1B). No nematodes disembarked (except 1 sample in which a single nematode disembarked) during the 12-day incubation period. The line inside the box plot is the median, the top of the box is the third quartile, the bottom of the box is the first quartile, the upper bar is the 90th percentile, the lower bar is the 10th percentile, and the white circle is an outlier. Bars indicate SD. ${ }^{*} \mathrm{P}<0.01,{ }^{* * *} \mathrm{P}$ $<0.0001$, A NOVA.

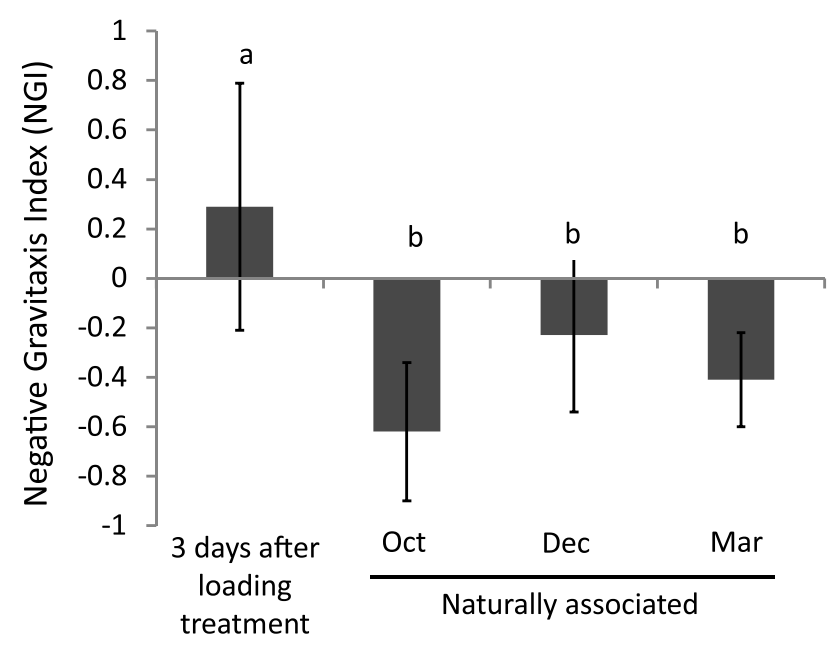

Fig. 5. Comparison of the gravitactic behavior of $C$. japonica $D L$ in different conditions. C. japonica DL were collected from $P$. japonensis 3 days after artificial loading with $C$. japonica followed by desiccation treatment $(\mathrm{N}=5)$ and from $\mathrm{P}$. japonensis collected during October, December, and the following $M$ arch ( $N=10,13$, and 10 , respectively). Bars indicate SD. Different letters indicate statistical differences by a Bonferroni/Dunn test $(P<0.05)$. 
of nymphs as well as a gravitactic behavioral change of $\mathrm{DL}$ on their carrier are important factors for disembarkation of C. japonica.

Humidity is one of the most important abiotic factors that affect nematode activity. It is also a useful cue for phoretic nematodes on their carrier because it indicates that they have reached their propagation site. A key disembarkation cue for Caenorhabditis remanei is humidity, and C. remanei DL readily disembark from their carriers in high humidity conditions (Baird, 1999). However, in our study, we found that high humidity was not a sufficient cue for $\mathrm{C}$. japonica DL disembarkation.

Caenorhabditis japonica is a bacterial-feeding nematode, and thus, we considered that the presence of $\mathrm{E}$. coli as a food source or habitat soil containing microbes, leaf litter, and plant roots might trigger nematode disembarkation. However, the presence of E. coli and soil did not stimulate disembarkation by $C$. japonica DL. When C. japonica propagates in the nest of $P$. japonensis, there are fresh egg carcasses and nymphal cadavers of $\mathrm{P}$. japonensis soon after hatching and $C$. japonica seems to feed on fresh nutrients from $P$. japonensis rather than bacteria (Okumura et al., 2013a). These previous observations and the present results suggest that the presence of bacteria is not a preferred cue for disembarkation by $C$. japonica DL.

In a previous study, very few DL were detected on mother insects after hatching (Okumura et al., 2013a). Immediately before hatching, the mother insect spreads symbiotic bacteria on the surface of the egg mass. The symbiotic bacteria on the egg mass are ingested by the $P$. japonensis nymphs while feeding on trophic eggs provided for the nymphs, which are used for nitrogen recycling in $\mathrm{P}$. japonensis (Hosokawa et al., 2010). Thus, we considered that a disembarkation cue may be provided by the symbiotic bacteria. A nother possibility was that hatching itself could trigger nematode disembarkation. Unexpectedly, however, almost no disembarkation was observed in the presence of symbiotic bacteria and hatching stimuli.

The most effective $\mathrm{DL}$ disembarkation cue was the presence of nymphs. Nymphs actively feed on drupes that they metabolize for their growth. Odors from the nymphs could be an important disembarkation cue ensuring the presence of food for C. japonica DL. Physical stimuli such as movements and/or sounds by the nymphs may also contribute to disembarkation. In the present study, we were not able to prepare $P$. japonensis females that were treated with neither hatching nor nymphal stimuli for comparison because of the limitation of $P$. japonensis numbers. Thus, we cannot deny the possibility that not only the presence of nymphs but also the combinations of incubation in high humidity, hatching stimuli, and the presence of nymphs, stimulate the disembarkation. There is also a possibility that a longer incubation period also stimulates disembarkation. In our preliminary observation, however, longer incubation in high humidity did not stimulate disembarkation very well.

The presence of nymphs enhanced disembarkation, but not all DL disembarked. Thus, a combination of symbiotic bacteria, hatching, the presence of nymphs, and/or the consequences of these phenomena may constitute the overall disembarkation cue, which indicates favorable conditions for $C$. japonica. We used reproductive diapause females because of the difficulty in preparing sufficient numbers of gravid females for the present study. In a preliminary study using gravid P. japonensis females, the disembarkation profile in high humidity conditions was similar to that with reproductive diapause P. japonensis females. However, we could not exclude the possible effect of the physiological condition of the mother insect from the present study.

We recently reported that nictating $C$. japonica $D L$ show a negative gravitactic behavior (Okumura et al., 2013c). T he negative gravitactic behavior of $D L$ appears to increase the opportunity to encounter its carrier insects wandering on the ground and could be useful for embarkation. In the present study, C. japonica DL artificially loaded onto $P$. japonensis for 3 days still showed the negative gravitactic behavior. On the other hand, DL from $\mathrm{P}$. japonensis collected in October, December, and March exhibited positive gravitaxis. New adults of $P$. japonensis emerge in late June to early A ugust. T he DL from P. japonensis in October, December, and March had been associated with P. japonensis for more than 2 months. The longer duration of the association may have altered the behavioral response to gravity. We do not know the mechanism that underlies this gravitactic change, but this behavioral change appears to be important for the disembarkation from their carrier. In the present study, we used not only naturally associated DL but also artificially loaded DL for negative gravitaxis experiments because it was difficult to obtain $\mathrm{P}$. japonensis with $C$. japonica DL immediately after nematode embarkation in the field. However, because artificially loaded DL were found at similar body parts of $P$. japonensis and were morphologically similar to naturally associated ones, our results are suggestive of the natural phenomenon in the field. Further study is necessary to elucidate the mechanisms of changes in gravitactic behavior under controlled conditions.

In our previous studies, we found that $C$. japonica forms a species-specific and female-host biased phoretic/necromenic association with $P$. japonensis 
(Okumura et al., 2013a, b; Y oshiga et al., 2013). The survivorship of $C$. japonica $D L$ is maintained at high levels over months on $\mathrm{P}$. japonensis while it is low without $\mathrm{P}$. japonensis (T anaka et al., 2010b, 2012). M oreover, our present results suggest that the disembarkation of $C$. japonica $\mathrm{DL}$ is controlled by complex mechanisms to ensure its association with $\mathrm{P}$. japonensis. These data indicate that the life cycles of $C$. japonica and $P$. japonensis are synchronized and $C$. japonica has sophisticated mechanisms to associate with $P$. japonensis.

\section{ACKNOW LEDGMENT}

The authors thank Ms Hiromi Mukai of Saga University for her assistance with the artificial hatching technique.

\section{LITERATURE CITED}

Baird, S. E. (1999) Natural and experimental associations of Caenorhabditis remanei with Trachelipus rathkii and other terrestrial isopods. Nematology 1, 471-475.

Hara, A. H., Lindegren, J. E. and Kaya, H. K. (1981) Monoxenic mass production of the entomogenous nematode, Neoplectana carpocapsae W eiser on dog food/agar medium. USDA/SEA, A dvanced A griculture T echnology. Western Series 16, Oakland, 8 pp.

Hosokawa, T., Kikuchi, Y., Nikoh, N., Meng, X. Y., Hironaka, M. and Fukatsu, T. (2010) Phylogenetic position and peculiar genetic traits of a midgut bacterial symbiont of the stinkbug Parastrachia japonensis. A pplied and Environmental Microbiology 76, 41304135.

Kiontke, K. (1996) The phoretic association of Diplogaster coprophila Sudhaus \& Rehfeld, 1990 (Diplogastridae) from cow dung with its carriers, in particular flies of the family Sepsidae. Nematologica 42, 354-366.

Kiontke, K. (2006) E cology of Caenorhabditis species. In: WormBook (The C. elegans Research Community ed.), http://www.wormbook.org.

Kiontke, K., Hironaka, M. and Sudhaus, W. (2002) Description of Caenorhabditis japonica $n . s p$. (Nematoda: Rhabditida) associated with the burrower bug Parastrachia japonensis (Heteroptera: Cydnidae) in Japan. Nematology 4, 933-941.

Liuzzi, V. C., Giancaspero, T. A ., Gianazza, E., Banfi, C., Barile, M. and Giorgi, C. D. (2012) Silencing of FAD synthase gene in Caenorhabditis elegans upsets protein homeostasis and impacts on complex behavioral patterns. Biochimica et Biophysica A cta 1820, 521-531.

Okumura, E., Ishikawa, Y., T anaka, R. and Y oshiga, T. (2013a) Propagation of Caenorhabditis japonica in the nest of its carrier bug, Parastrachia japonensis. Zoological Science 30, 174-177.

Okumura, E., T anaka, R. and Y oshiga, T . (2013b) Speciesspecific recognition of the carrier insect by dauer larvae of the nematode Caenorhabditis japonica. The Journal of Experimental Biology 216, 568-572.

Okumura, E., T anaka, R. and Y oshiga, T. (2013c) Negative gravitactic behavior of Caenorhabditis japonica dauer larvae. The Journal of Experimental Biology 216, 14701474.

Poiner, G. O. (1975) Entomogenous nematodes. A manual and host list of insect nematode association. E. J. Brill, Leiden, $317 \mathrm{pp}$.

Sudhaus, W. and Schulte, F. (1989) Rhabditis (Rhabditis) necromena sp. $n$. (Nematoda: Rhabditidae) from south A ustralian diplopoda with notes on its siblings $R$. myriophila Poinar, 1986 and R. caulleryi Maupas, 1919. Nematologica 35, 15-24.

T anaka, R., Okumura, E. and Y oshiga, T. (2010a) A simple method to collect phoretically active dauer larvae of Caenorhabditis japonica. Nematological Research 40, 712.

T anaka, R., Okumura, E. and Y oshiga, T. (2010b) Survivorship of Caenorhabditis japonica dauer larvae naturally associated with shield bug, Parastrachia japonensis. Nematological Research 40, 47-52.

T anaka, R., Okumura, E., K anzaki, N. and Y oshiga, T. (2012) Low survivorship of dauer larva in the nematode Caenorhabditis japonica, a potential comparative system for a model organism, C. elegans. Experimental Gerontology 47, 388-393.

T sukamoto, L. and Tojo, S. (1992) A report of progressive provisioning in a stink bug, Parastrachia japonensis (Hemiptera: Cydnidae). Journal of Ethology 10, 21-29.

Winston, P. W. and Bates, D. H. (1960) Saturated solution for the control of humidity in biological research. Ecology 41, 232-237.

Y oshiga, T ., Ishikawa, Y., T anaka, R., Hironaka, M. and Okumura, E. (2013) Species-specific and female hostbiased ectophoresy in the roundworm Caenorhabditis japonica. Naturwissenschaften 100, 205-208.

Received: November 19, 2012 